patient per month (PPPM) medical costs were calculated 12 months after the PAM assessment date. Costs were constructed in 4 areas: total, ED, inpatient and pharmacy. Using Ordinary Least Squares Regression, each cost measure was regressed on PAM level (PAM 1 [reference group] vs. PAM 2, PAM 3 , and PAM 4); adjusting for the following covariate measures; demographics and a severity of illness. We performed statistical analyses on logged PPPM medical costs, but report comparisons in absolute dollars (2011). Results: Compared to those with PAM 1 scores, those with PAM 3 scores had lower total costs (beta coefficient $=-\$ 849 ; P=.0004)$, ED costs (beta coefficient $=-\$ 22 ; P=.02)$ and inpatient costs (beta coefficient $=-\$ 500 ; P=.03$ ). Conclusions: Among a population with hypertension; adults with higher PAM scores (PAM 3) had significantly lower total costs, ED costs and inpatient costs, compared to patients with lower PAM scores (PAM 1).

Keywords: Patient activation; Medical costs

$$
\text { doi:10.3121/cmr.2014.1250.ps1-23 }
$$

PS1-38:

Building a Patient-centered Medical Home: Patient Perspectives from a Survey in an Integrated Healthcare System

Samuel Forjuoh ${ }^{1}$; Sarah Miller ${ }^{1}$ Michael Reis ${ }^{1}$

${ }^{1}$ Scott \& White Healthcare

Background/Aims: As healthcare evolves to meet growing demand, it is crucial to maintain quality patient-centered care. In our quest to build a viable Patient-Centered Medical Home (PCMH) in our ambulatory care setting, we conducted this study to assess our patients' perspectives on patientcenteredness in our integrated healthcare system. Methods: Surveys were administered to all consenting English-speaking patients and accompanying relatives ages $=18$ at 4 Family Medicine clinics. The questionnaire consisted primarily of Likert-type items with 5 choices ranging from strongly agree to strongly disagree on relationships within healthcare, communication with providers, access to providers, and coordination of care, along with basic demographics. Results: Of 316 patients approached, 204 (64.6\%) returned completed surveys. Mean age was 47.4 ( $\mathrm{SD}=16.0$; range 18-89); $67 \%$ were female, $84.4 \%$ white, $11.8 \%$ black, and $12.8 \%$ Hispanic. Majority were married $(57.0 \%)$, employed $(60.2 \%)$, had college education $(69.4 \%)$, and reported an annual income of $<\$ 60,000(68.7 \%)$. The vast majority expressed believe that their healthcare team treats them with courtesy and respect $(96.1 \%)$, trust their healthcare team with their medical care $(93.6 \%)$, feel their healthcare provider communicates with them clearly using understandable language $(95.6 \%)$ and listens to their questions, answering them directly (96\%). Also the vast majority believe they play an active role in their healthcare $(95.5 \%)$ and think it is important to be proactive in their healthcare $(97 \%)$. Comparatively lower percentages of positive responses were noted when asked if the patient would like to use the Internet for filling prescriptions, making appointments, or communicating with their healthcare team $(52 \%)$, if their provider inquires about their health goals $(61.9 \%)$, and if their provider takes their health goals into account when developing a treatment plan (69.4\%). Conclusions: In order to move towards a more PCMH, providers need to talk to patients about their health goals and incorporate them in developing a treatment plan. Since patients seem willing to take a proactive role in their healthcare and already trust their healthcare team, a PCMH model appears to be an attainable goal in our setting. Keywords: Patient-centered medical home; Patient perspectives

$$
\text { doi:10.3121/cmr.2014.1250.ps1-38 }
$$

\section{Statistical and Research Methods}

B4-2:

Risk Engine Evaluation Software: An Analytics Platform for Individualized Guidelines

Evelyn Whitlock ${ }^{1}$; Tuan Dinh ${ }^{2}$

${ }^{1}$ Kaiser Permanente Northwest; ${ }^{2}$ Archimedes

Background/Aims: With the recent explosion of medical data, it is necessary to develop software tools to support the process of evidence review and synthesis that is fundamental to Evidence-Based Medicine. Furthermore, as predictive models are being increasingly integrated into clinical decision making and personalized guidelines for individual patients, there is an even greater need for a practical tool that enables development, validation, and uncertainty quantification of models in a robust, automatic and efficient way. Methods: We developed Risk Engine Evaluation Software, a software platform that enables users to compare and synthesize evidence from multiple data sources, to build robust and accurate predictive models to estimate and stratify disease risks, to validate risk equations and to quantify the accuracy of a predictive model in different subpopulations. Results: We applied the tool to data on cardiovascular outcomes from a large number of trials, observational studies and electronic medical records, including Framingham Heart Study, Atherosclerosis Risk in Communities Study, Cardiovascular Health Study, and ALLHAT. We demonstrated that users can evaluate the performance of predictive models for different subpopulations in real time. Several metrics were used for model evaluation: cumulative incidence, calibration plots, receiver operating curve (ROC) and net reclassification index. The software also allows users to generate and then assess models for risks of MI, stroke or heart failure, for a predefined subpopulation from selected datasets. Conclusions: Risk Engine Evaluation Software proves to be a useful tool for advancing the development and application of predictive modeling in medicine.

Keywords: Risk model; Individualized guidelines

doi:10.3121/cmr.2014.1250.b4-2

B4-3:

Is There Evidence of Non-response Bias for Survey-based Estimates of Urinary Incontinence?

Haiyan Sun ${ }^{1}$

${ }^{1}$ Geisinger Health System

Background/Aims: Non-response is an important potential source of bias in survey research. It is usually difficult to obtain meaningful information on non-respondents to adequately assess bias on estimates of population statistics, especially when the survey involves sensitive or intrusive questions. We combined Electronic Health Record (EHR) and survey data to assess the impact of non-response on estimates of urinary incontinence (UI) prevalence. We used data from the baseline survey of the General Longitudinal Overactive Bladder Evaluation - Urinary Incontinence (GLOBE-UI), a population-based study of the natural history of UI in women $=40$ years of age. We conducted the survey on a random sample of 7,125 Geisinger Clinic Primary Care patients. The response to the baseline survey was $57 \%$. We also used electronic health records (EHR) data to obtain demographic, care utilization, health behavior, social history, and clinical information on all eligible respondents of the GLOBE-UI survey. Methods: We complete a two-stage model. First, we used logistic regression to assess baseline characteristics associated with response status. The predicted probabilities were used as a weight in the second stage model. UI status was defined for each response based on survey data. An Inverse Probability weighting (IPW) logistic regression model was applied to adjust non-response bias and to determine prevalence of UI based on baseline risk factors associated with UI status, including baseline characteristics, health utilizations, social and educational history, and comorbidities. The non-response adjusted estimate of prevalence was compared with prevalence obtained from respondents. Results: Age at survey, smoking, marital status, number of outpatient encounters per year, CHF, dementia, and severe liver disease were significantly associated with response status and UI status. Adjusting for non-response bias resulted in a reduction of the UI prevalence estimate from an observed value of $31 \%$ to an adjusted value of $28 \%$ for those 60 and older. The observed and adjusted estimates were not different for younger respondents. Conclusions: Non-response bias in women with UI is limited. It may lead to a marginal overestimate of UI prevalence for older patients. This could be explained by the non-response group being relatively younger. Keywords: Non-response bias; IPW logistic regression model doi:10.3121/cmr.2014.1250.b4-3 